\title{
An Augmented Reality based Car Parking Game
}

\author{
Sameer Virnodkar \\ Department of Information \\ Technology Engineering \\ Vidyalankar Institute of \\ Technology \\ Mumbai, India
}

\author{
Anuj Khairnar \\ Department of Information \\ Technology Engineering \\ Vidyalankar Institute of \\ Technology \\ Mumbai, India
}

\author{
Moxesh Mehta \\ Department of Information \\ Technology Engineering \\ Vidyalankar Institute of \\ Technology \\ Mumbai, India
}

\author{
Chintan Shah \\ Department of Information Technology Engineering \\ Vidyalankar Institute of Technology \\ Mumbai, India
}

\begin{abstract}
Gaming on mobile phones has been gaining popularity since its advent in 1994 with Hagenuk launching its MT-2000 loaded with Tetris.[1] Since then, as technology advanced, gaming on mobile phones moved from black \& white to color and eventually to graphically-intense 3D.

In this paper, we propose an android mobile game based on augmented reality, which features a parking lot where a player controls a car, which he must move a from an initial state to a pre-defined parking spot without getting into contact with any obstacles. The difficulty increases with each level. As the game implements Augmented Reality technology, the game play incorporates the surrounding environment of the player, i.e. Floor, table, bench, desk, etc. providing a more interactive mobile-gaming experience. 'pARking - an AR based Game' is an Android Gaming application which will enable the end user to experience the new emerging technology called 'Augmented Reality' (shortened as AR).
\end{abstract}

\section{General Terms}

Augmented Reality, Game, Car, Android.

\section{Keywords}

pARking, mobile game, parking spot, obstacle.

\section{INTRODUCTION}

Mobile games have always been the primary and most reliable way of entertainment for people since the mid-90s. The gaming industry has always been on a growth curve when revenue generation is considered. In its revolution from a simple Tetris game with a high graphics, fully customizable FIFA, everything that a gamer can imagine has changed; right from the visuals to the controls and from the sound of the story of a game. But, there was always a gap in between the real world and the virtual game world, if eliminated, could change the gaming world forever. Introduction of Augmented Reality technology into the gaming industry brought about that change.

'pARking - an AR based Game', will provide the end user a very sweet and simple way to experience this new AR technology through a game, which can be played on their Android Device, for a great price of zero i.e. for free of cost. The player just needs to download and install the game 'pARking' from Google Play Store and enjoy Augmenting the entire gaming environment on any surface like a table, floor or to chair, bed, on the street, in the garden or on any possible surface.

Once the user opens the app, selects the level and starts the game, it will automatically open the camera through the game up and ask for the required Target Marker which is simply an image on a piece of paper (printout) whose softcopy will be provided by us. As soon as the user scans that Image Target, the entire game layout or environment will be augmented on the place of that image. Now, the user can start playing the game. The user will be provided a car who will navigate through the parking lot to park the car at a specified position.

While navigating, if the car collides on any of the obstacles, then automatically the game will be over. In this way, the 'pARking', will be available to users with multiple levels in it, so that difficulty level also increases as the levels progress. This makes 'pARking' a full-fledged AR based Android Game which will be available on Google Play.

\subsection{Usefulness}

It will change the perspective of the gamers who have a standard or normal playing experience of how a game is played.

Use of Augmented Reality concept will enable the players to interact with their surroundings in real time through the game.

It will be helpful for companies to develop more such games with added functionality.

In the rest of the paper, we first review the related work of the game in the Section 2. We then state the Aim and Objectives of this game in Section 3. The Section 4 describes the current scenario in the mobile phone gaming industry, explaining the scarcity of AR based mobile games in the market. The proposed system is then described in Section 5. The next section, Section 6 outlines the scope of the system, followed by the system methodology in the Section 7 . Finally, we draw conclusion and give references.

\section{RELATED WORK}

Previous work has been referred, which has helped us to understand and apply the Augmented Reality concept for this game.

In the year 2006, Ching-Sheng Wang, Shih-Hui Hung and Ding-Jung Chiang [1], have written a paper proposing a system implementing Augmented Reality for navigation purposes. The system can recognize multiple marker-less 
images from a long distance and provide navigation information related to it, thus improving upon the traditional barcodes and QR codes, which are ineffective if not scanned from a close distance, and are difficult to scan in crowded places. It explains the advantage of using multiple marker-less image targets, as compared to ones with conventional barcode and QR codes.

In the year 2006, Claudio Kimer, Ezequiel R. Zorzal, and Tereza G.Kirner [2], have written a paper featuring case studies on development of games using Augmented Reality. It includes case studies of five games which are built using the ARToolKit. The games are: Three Dimensional Jigsaw puzzle, Tic-tac-toe, game of Words, Musical games and Magic cubes. ARToolKit which is used to build these games, is an Open Source software that is available free of cost, which can be downloaded over the internet.

\section{AIM AND OBJECTIVE}

\subsection{Aim}

Since Augmented Reality is a new and emerging technology, non-tech-savvy people or who are not related to computer science field, are unable to relate to it or they are not aware of this technology or some people are just aware but have not experienced this technology of AR.

So, the aim of this game 'pARking - an AR based Game' is to familiarize people with this new AR technology and provide them a very sweet and simple way to use this new AR technology by simply playing a Game on their Android Device, without spending a single rupee.

\subsection{Objective}

Also, our objective is to develop a game that can be played by people of any ages, providing a more interactive gaming experience by incorporating augmented reality. We will also be implementing a concept of virtual buttons in Augmented Reality.

\section{PROBLEM STATEMENT}

Existing mobile games are not that interactive; they are just games which are to be played on the mobile device. But, our game will be based on a modern technology called Augmented Reality which will make the mobile gaming experience more interactive and will be augmented on the Real-World objects like any surface such as Table, Floor, bench etc.

Also, various car parking games are available in the market, but the problem with those games is that they are not that immersive. There is also a scarcity of AR Based Games in the market, hence the field of AR based gaming is almost untouched. Due to the above factors, people are not quite aware of the Augmented Reality technology. We will try to familiarize the audience with this technology through this game.

\section{PROPOSED SYSTEM}

The proposed system is an Augmented Reality based parking game which includes augmenting of virtual 3D models into real world which totally changes the experience and feel of playing games.

The system will include number of car models where a user must select any one and start the game. As the game starts, the user will have to move from the initial position to the predefined parking space without hitting any of the existing obstacles in between. And if the user accomplishes to do so, the user will be able to jump into the next level else will have to repeat the same level until it gets completed successfully.

The layout of the game will be simple and easy to understand. People of all ages, from children to elders will be able to play the game without any difficulty.

\section{SCOPE}

The scope of this game is as below:

- It will provide a much more interactive and appealing game which will entertain and amaze the users with its augmented reality functionality.

- The game will include various levels, each with an increasing complexity where a user will have to reach from the start point to the parking end point without clashing into any obstacles.

- The gamer will be able to into any level in between once it is clear or is successfully completed.

- The game will be a car parking game which will be developed using augmented reality.

- The game will feature a parking lot, and the user will navigate through the parking lot to park the car at a specified position.

- The game will feature multiple levels, with the difficulty increasing as the game progresses.

\section{METHODOLOGY}

Our game 'pARking — an AR based Game' is an Augmented Reality based Android Gaming application which will enable the end user to experience the new emerging technology called Augmented Reality (shortened as AR). The term AR in the game name 'pARking' itself suggests about this technology. Our game 'pARking - an AR based Game' will provide the end user a very sweet and simple way to use this new AR technology by simply playing a game on their Android Device, for a great price of zero i.e. for free of cost.

The player just needs to download and install the game 'pARking' from Google Play Store and enjoy Augmenting the entire gaming environment on any surface like a table, floor or on chair, bed, on the street, in the garden or on any possible surface.

The proposed car parking game consists of total ten levels, each with an increased complexity than the previous one, i.e. complexity will be increasing as the levels progress as shown in fig. 7.1.

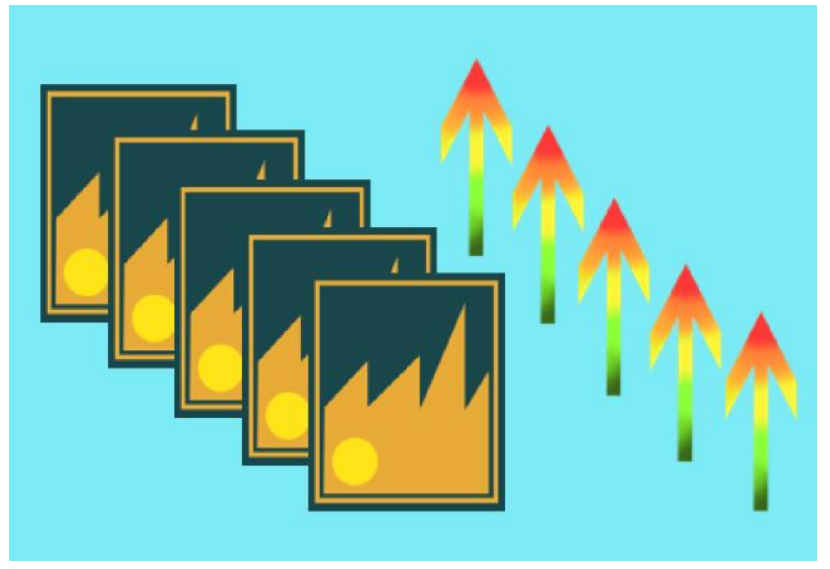

Figure 1: Complexity increases with levels

In each level, the user will be provided a car who will navigate through the parking lot to park the car at a specified 
position as shown in fig.7.2. While navigating, if the car collides on any of the obstacles, then automatically the game will be over. In this way, the game 'pARking' will be available to users with multiple levels in it, so that difficulty level also increases as the levels progress. This makes 'pARking' a full-fledged AR based Android Game which will be available on Google Play.

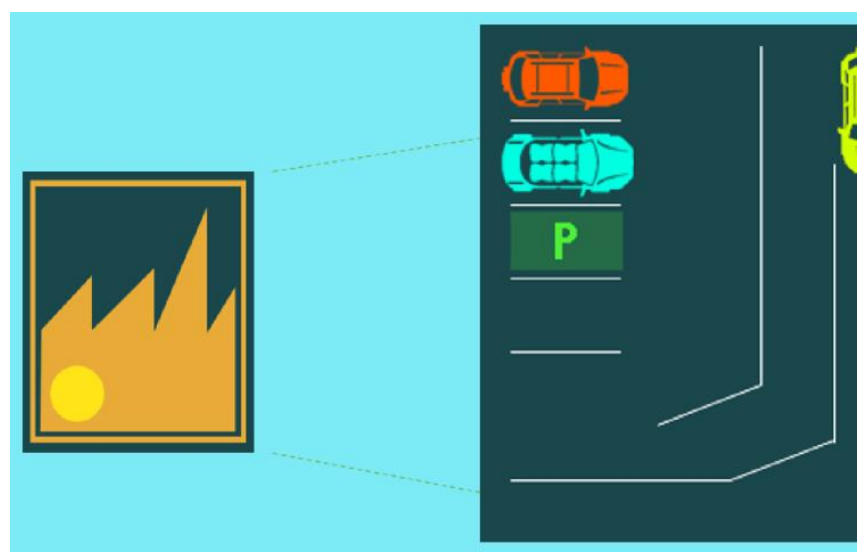

Figure 2: Sample Level Map

\section{CONCLUSION}

This paper presents an Augmented Reality based game named 'pARking' built using Unity 3D software and Vuforia that changes the perception of how game is played. As the game includes virtual 3D model with various level of complexity at every stage, it will provide rich experience and feel of playing games. We plan to add multiplayer support and leaderboards in the future. These will make the gameplay more interesting and increase the user base.

\section{REFERENCES}

[1] Bowman, M., Debray, S. K., and Peterson, L. L. 1993. Reasoning about naming systems.

[2] Ding, W. and Marchionini, G. 1997 A Study on Video Browsing Strategies. Technical Report. University of Maryland at College Park.

[3] Fröhlich, B. and Plate, J. 2000. The cubic mouse: a new device for three-dimensional input. In Proceedings of the SIGCHI Conference on Human Factors in Computing Systems

[4] Tavel, P. 2007 Modeling and Simulation Design. AK Peters Ltd.

[5] Sannella, M. J. 1994 Constraint Satisfaction and Debugging for Interactive User Interfaces. Doctoral Thesis. UMI Order Number: UMI Order No. GAX9509398., University of Washington.

[6] Forman, G. 2003. An extensive empirical study of feature selection metrics for text classification. J. Mach. Learn. Res. 3 (Mar. 2003), 1289-1305.

[7] Brown, L. D., Hua, H., and Gao, C. 2003. A widget framework for augmented interaction in SCAPE.

[8] Y.T. Yu, M.F. Lau, "A comparison of MC/DC, MUMCUT and several other coverage criteria for logical decisions", Journal of Systems and Software, 2005, in press.

[9] Spector, A. Z. 1989. Achieving application requirements. In Distributed Systems, S. Mullender 